University of Nebraska - Lincoln

DigitalCommons@University of Nebraska - Lincoln

2013

\title{
Ab-initio study of free standing TiO2 clusters: Stability and magnetism
}

\author{
T.H. Rana \\ LNM Institute of Information Technology \\ Pankaj Kumar \\ Kendriya Vidhyalaya, panksamrat@gmail.com \\ Ashok K. Solanki \\ University of Nebraska-Lincoln, asolanki2@unl.edu \\ Ralph Skomski \\ University of Nebraska-Lincoln, rskomski2@unl.edu \\ Arti Kashyap \\ University of Nebraska-Lincoln, akashyap@Inmiit.ac.in
}

Follow this and additional works at: https://digitalcommons.unl.edu/physicsskomski

Rana, T.H.; Kumar, Pankaj; Solanki, Ashok K.; Skomski, Ralph; and Kashyap, Arti, "Ab-initio study of free standing TiO2 clusters: Stability and magnetism" (2013). Ralph Skomski Publications. 82.

https://digitalcommons.unl.edu/physicsskomski/82

This Article is brought to you for free and open access by the Research Papers in Physics and Astronomy at DigitalCommons@University of Nebraska - Lincoln. It has been accepted for inclusion in Ralph Skomski Publications by an authorized administrator of DigitalCommons@University of Nebraska - Lincoln. 


\title{
Ab-initio study of free standing $\mathrm{TiO}_{2}$ clusters: Stability and magnetism
}

\author{
T. H. Rana, ${ }^{1,2}$ Pankaj Kumar, ${ }^{2,4}$ A. K. Solanki, ${ }^{3}$ R. Skomski, ${ }^{4}$ and A. Kashyap ${ }^{2, a)}$ \\ ${ }^{1}$ The LNM Institute of Information Technology, Jaipur, Rajasthan, India \\ ${ }^{2}$ School of Basic Science, IIT Mandi, Mandi, Himachal Pradesh, India \\ ${ }^{3}$ Malviya National Institute of Technology, Jaipur, Rajasthan, India \\ ${ }^{4}$ Department of Physics and Astronomy and NCMN, University of Nebraska, Lincoln, Nebraska, USA
}

(Presented 18 January 2013; received 9 November 2012; accepted 16 January 2013; published online 11 April 2013; corrected 24 April 2013)

\begin{abstract}
We report the structural behavior of nanoscale Titanium Dioxide $\left(\mathrm{TiO}_{2}\right)$ clusters as well as their magnetic properties by varying the cluster size with the help of ground state geometries. The clusters of atomic scale rutile $\left(\mathrm{TiO}_{2}\right)_{\mathrm{n}}$, where $n=1-11$, have been considered and geometrically stabilized through the Density Functional Theory as implemented in Vienna ab-initio Simulation Package. It is being observed that as the size of cluster increases from $n=2$ to 11 , the total energy decreases. The results of formation energy reveal the fact that as the cluster grows, it moves towards the stability and it is observed that $n=11$ is the most stable structure. The stabilized clusters are different in geometries and co-ordination numbers. Finally, all the clusters have been investigated with self consistent treatment of spin orbit coupling for magnetism studies. The magnetic properties of free clusters depict oscillatory behavior for magnetic moment with respect to the cluster size. (C) 2013 American Institute of Physics. [http://dx.doi.org/10.1063/1.4799616]
\end{abstract}

\section{INTRODUCTION}

Since last decade, the research of free transition metal oxide nanoclusters has received great attention and has become the subject of much interest. A nanocluster is nothing but a nanometer sized particle made up of equal subunits. The structural and magnetic properties of these clusters are entirely different from bulk value due to high surface to volume ratio as well as electron confinements with boundary conditions. The crystalline $\mathrm{TiO}_{2}$ exists in three phases: rutile (tetragonal, $\mathrm{P} 4_{2} / \mathrm{mnm}$ ), anatase (tetragonal, $\mathrm{I} 4_{1} / \mathrm{amd}$ ), and brookite. Titanium dioxide $\left(\mathrm{TiO}_{2}\right)$ is widely investigated by researchers because of the applications such as use in solar cell, paints, cosmetics, etc. ${ }^{1-10} \mathrm{We}$ are interested in rutile $\left(\mathrm{TiO}_{2}\right)_{n}$ nanoparticles for several reasons. They are structurally stable at nanoscale and used for astrophysical application in dust formation of oxygen rich stars. ${ }^{11}$ These nanoclusters also have potential applications in spintronics and optics due to room temperature mangnetism. ${ }^{12-14} \mathrm{~A}$ wide plethora of reported studies already exist on the spectroscopic analysis of $\mathrm{TiO}_{2}$ metal dioxide clusters giving information about the structure and bonding in clusters. ${ }^{15}$ It is being addressed the electronic structure information about $\left(\mathrm{TiO}_{2}\right)_{\mathrm{n}}$ clusters with $n=1-10$ using photoelectron spectroscopy (PES) and the magnetism for anatase phase clusters has been observed experimentally by Wei et al. ${ }^{16}$ Chiodo et al. ${ }^{17}$ and Mo et al. ${ }^{18}$ have studied the electronic, structural, and optical properties of titanium dioxide clusters employing ab-initio calculations. Relative stability of $\left(\mathrm{TiO}_{2}\right)_{n}$ cluster for different symmetries was studied by Albaret et al. using Density Functional Theory (DFT). ${ }^{19}$ The neutral $\left(\mathrm{TiO}_{2}\right)_{n}$ clusters with $n=1-6$ were reported by Jeong et al. using the

\footnotetext{
${ }^{\text {a) }}$ Author to whom correspondence should be addressed. Electronic mail: arti.kashyap@gmail.com
}

DFT approach. ${ }^{20}$ To sum up, during the last ten years, there is substantial growth in generation and understanding the properties of these clusters, which involves varying size of clusters, change in geometry and physical properties such as magnetism. Due to varying geometries, it is complicated to find global minima of stabilized cluster with same energy being totally dependent upon approximations. ${ }^{21}$ The smallest monomer, the $\mathrm{TiO}_{2}$ cluster model was characterized by $\mathrm{C}$. Tsipis et al. and onwards the $\left(\mathrm{TiO}_{2}\right)_{2}$ has been taken as unitary building block. ${ }^{22,23}$ Implementing first principle calculation, with main attention devoted to their stability study $\left[\left(\mathrm{TiO}_{2}\right)_{n}, n=1-4\right]$ (Ref. 24) and for larger clusters the $\left(\mathrm{TiO}_{2}\right)_{n}$ nanoparticles with $n=10-16$, an interesting oddeven oscillation in the structural features has been observed. $^{25}$

\section{METHODOLOGICAL FRAMEWORK}

\section{A. System investigated}

In the present paper, we have concentrated on various geometries of different clusters of $\left(\mathrm{TiO}_{2}\right)_{n}[n=2-11]$ within the ab-initio first principle approach using Vienna ab-initio simulation package (VASP) [5.2] code. The size of clusters varies from $n=2-11$ and each cluster was stabilized with lowest energy. Our main interest has been to look for the stabilized geometries of $\mathrm{TiO}_{2}$ clusters. The geometries of stable clusters are comparable to Zhang et al. ${ }^{26}$ The dependence of the induced magnetism on symmetry, structural geometry, and coordination number in all these clusters is the primary focus in this study. The aim is not only the atomic and structural point of view but also to observe how the magnetic moment varies as clusters' size varies from $n=2$ to $n=11$ depicting magnetism as a function of cluster size. As the principal sources of magnetism in atoms, clusters, and solids are the currents associated to the electronic motion (orbital magnetism) and the 
intrinsic angular momentum of the electrons (spin magnetism), the variation taken into account is fundamental for studying the same.

\section{B. Computational scheme}

The spin-polarized calculations are performed using VASP $^{27-29}$ with a sufficiently high cut off energy of $400 \mathrm{eV}$ and k-space integration at $\Gamma$-point only using Perdew-BurkeErnzerhof (PBE) for the exchange and correlation potential. The unit cell of rutile $\mathrm{TiO}_{2}$ is taken to be tetragonal (P42/mnm) with space group 136 and lattice parameters $a=4.594 \AA$ and $c=2.958 \AA$; subsequently, clusters of varying size are formed using the supercell approach. A spacing of $\sim 9 \AA$ has been maintained between two periodic clusters to avoid any interaction or correlation effect. The clusters vary with respect to the number of constituent atoms starting from 6 to 33 atoms, thereby the biggest being approximately $4 \mathrm{~nm}$ in size. The structural relaxation using $C G$ (conjugate gradient) algorithm is allowed for all configurations of the clusters. These geometries were optimized with converged force being $0.001 \mathrm{eV} / \AA$. In the present paper, most stable structures are discussed for the singlet state and the excitation occurs from $p$ orbital of oxygen to un-occupied $d$ orbital of titanium. ${ }^{30}$ For each system, the calculations have been performed following a two steps approach. First, the scalar relativistic nonspin polarized calculations have been performed using the generalized gradient approximation as the exchange and correlation approximations to find the minimum energy structure of the cluster by relaxing the cluster. Using this energy minimized nonmagnetic structure; we calculated the magnetic properties of clusters by performing the scalar relativistic spin polarized calculations. We used gaussian braodening in the integration of densities of states with sigma equal 0.2 , mimicking finite temperature effects. The magnetic moment of the cluster is not integer, while it is expected to have integer multiplicity at zero temperature.

\section{RESULTS AND DISCUSSION}

We examined the magnetization of $\mathrm{TiO}_{2}$ clusters as function of their size. The nanoclusters of rutile $\left(\mathrm{TiO}_{2}\right)_{n}$, where $n=2$ to 11 , have been dealt using PBE exchangecorrelation potential total energy is calculated for every size of the cluster under these approximations of potential and from that the formation energy per molecule is also calculated.

\section{A. Structural ground state geometries and cluster size dependence energetics}

The formation energy for a given structure nanocluster of $\left(\mathrm{TiO}_{2}\right)_{n}$, consisting of $n$ molecules of $\mathrm{TiO}_{2}$ can be given as ${ }^{31}$

$$
\mathrm{E}_{(\text {form })}=1 / \mathrm{n}\left\{\mathrm{E}\left[\mathrm{Ti}_{\mathrm{n}} \mathrm{O}_{2 \mathrm{n}}\right]\right\}-\mathrm{E}\left[\mathrm{TiO}_{2}\right] \text {, }
$$

where $\mathrm{E}\left[\mathrm{Ti}_{\mathrm{n}} \mathrm{O}_{2 \mathrm{n}}\right]$ is the total energy for the system and $\mathrm{E}\left[\mathrm{TiO}_{2}\right]$ is the energy of an isolated $\mathrm{TiO}_{2}$ molecule. We calculated formation energies for free $\left(\mathrm{TiO}_{2}\right)_{\mathrm{n}}$ clusters and the total energy curves with different approximations. Each cluster model has specific ground state geometry with lower

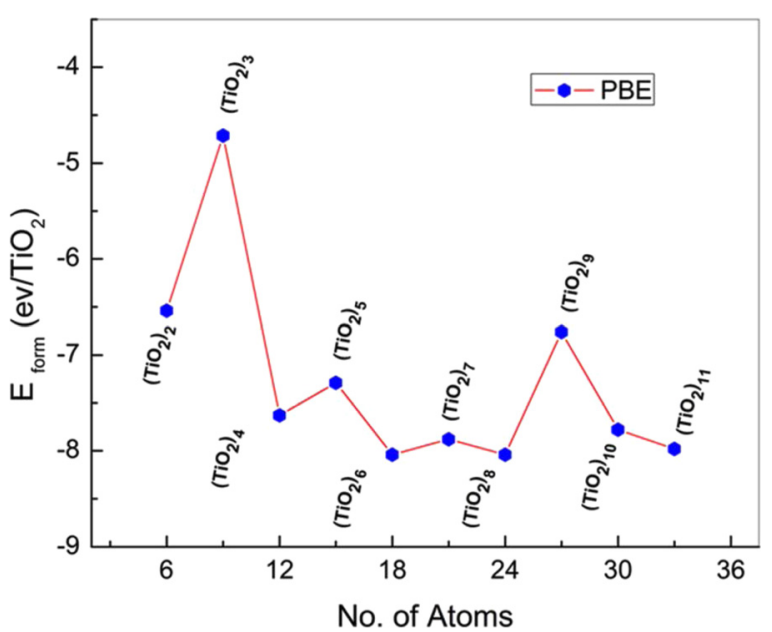

FIG. 1. Formation energy per $\mathrm{TiO}_{2}$ molecule as a function of cluster size.

energy. The nature of formation energy as a function of atomic size of cluster is shown in Fig. 1, which illustrates that, if the cluster size decreases, the total ground state energy increases towards positive. ${ }^{31}$ This happens so because when we move towards smaller nanoclusters, the surface to volume ratio becomes high. The surface atoms have a lower coordination in smaller cluster and average number of bond is lower. The surface energy contributes to the total ground state energy and becomes high in smaller cluster. If we take $\left(\mathrm{TiO}_{2}\right)_{\mathrm{n}}$ nano clusters, where $n=3,5,7,9,11$, they formed the ionic nature and other clusters, where $n=2,4,6,8,10$, had covalent nature so even number of $n$ clusters are more stable than odd number of $n$ clusters. In general, these all tend to form either ring or rodlike compact structures. Fig. 2 expresses the ground
-Titanium

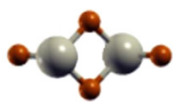

$\mathrm{Ti}_{2} \mathrm{O}_{4}$ Cluster
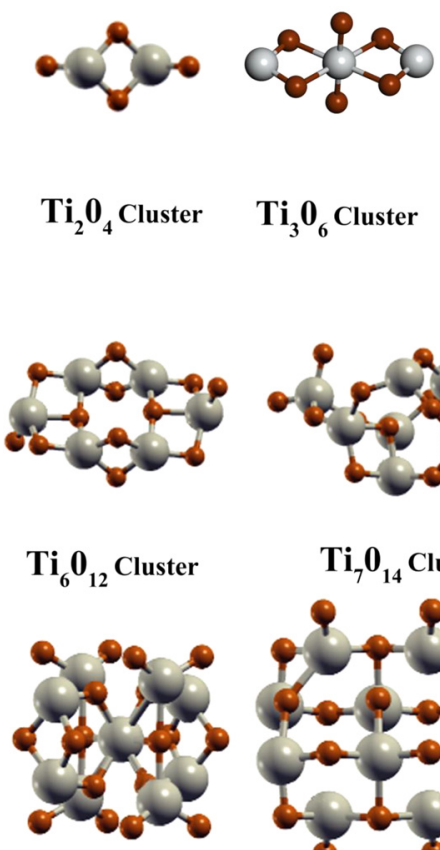

$\mathrm{Ti}_{9} \mathbf{0}_{18}$ Cluster
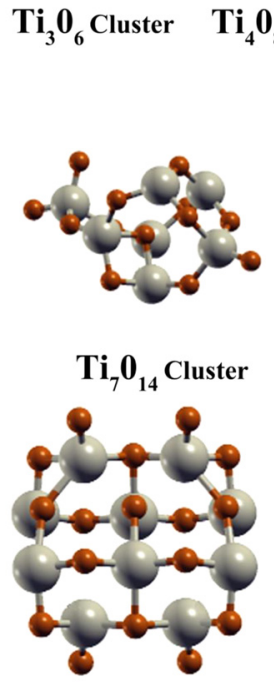

$\mathrm{Ti}_{10} \mathbf{0}_{20}$ Cluster
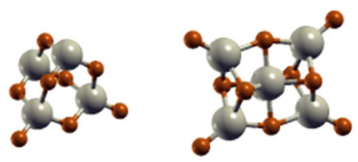

\section{-Oxygen}

$\mathrm{Ti}_{4} \mathrm{0}_{8}$ Cluster $\quad \mathrm{Ti}_{5} \mathrm{O}_{10}$ Cluster

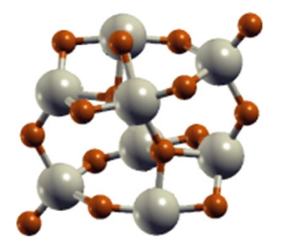

$\mathrm{Ti}_{8} \mathrm{O}_{16}$ Cluster

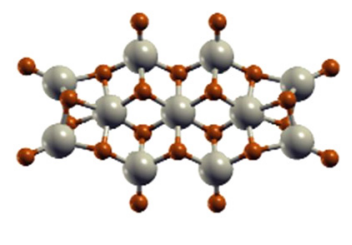

$\mathrm{Ti}_{11} \mathbf{0}_{22}$ Cluster
FIG. 2. Ground state geometries of stable clusters. 


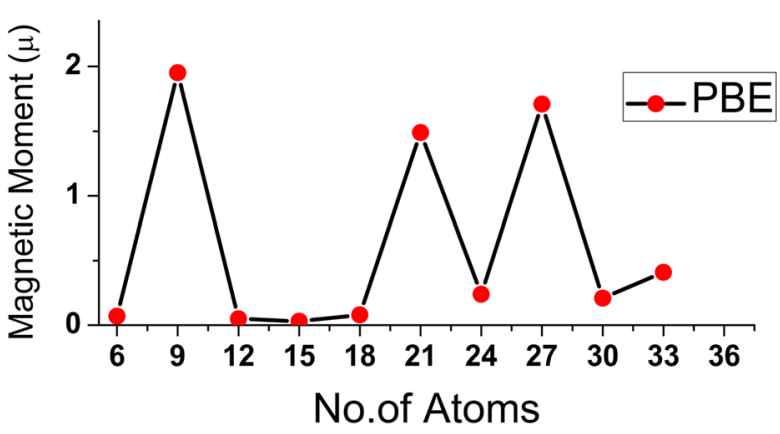

FIG. 3. Magnetic moment of free clusters as a function of atomic size using different approximations.

state geometries of free stable clusters having minimum energy. The $\left(\mathrm{TiO}_{2}\right)_{\mathrm{n}}$ cluster models in figure divulge the stability of clusters in singlet ground state because of transition from p-orbital of an oxygen to d orbital of titanium when they formed clusters. Titanium is a transition metal with an atomic configuration of $(3 d)^{2}(4 s)^{2}$ outside the argon core and oxygen with $(1 s)^{2}(2 s)^{2}(2 p)^{4}$ lead to $s-p$ - $d$ hybridization, thereby determining the structure of the cluster. The orbital compositions seem to be different in each cluster per size variation. ${ }^{32}$

\section{B. Magnetism in nanoclusters}

\section{Size-dependence of total magnetic moments}

The occurrence of magnetism in nanoclusters depends upon shape and structures of the nanogeometry. ${ }^{32}$ The magnetism as observed in these different sets of nanoclusters of $\mathrm{TiO}_{2}$ shows variations with respect to change in the size of the cluster. We have presented the magnetic moments of free clusters, which show the oscillatory behavior as shown in Fig. 3 with respect to the size of clusters reaching to $4 \mathrm{~nm}$ and total number of atoms in cluster varies from 6 to 33 . Figure 3 shows the variation of magnetic moment of the cluster as function of its size. The fluctuation of the moments is clearly observed. Local magnetic moments depend strongly on the arrangement of nearest neighbor atoms in the cluster. The magnetic moments oscillate with a maximum of $1.9473 \mu_{\mathrm{B}}$ for $\mathrm{Ti}_{3} \mathrm{O}_{6}$ clusters and a minimum of $0.0337 \mu_{\mathrm{B}}$ for $\mathrm{Ti}_{5} \mathrm{O}_{10}$ clusters and fluctuate in between these two extremities for rest of the configurations of the clusters using the PBE approximation. The origin of the oscillations in odd number of $n$ and even number of $n$ clusters can be explained from the nature of chemical bonding. The odd number of $\mathrm{n}$ clusters has more surface atom with lesser coordination resulting in higher net magnetic moment as compared to even number of $n$ clusters except $\mathrm{Ti}_{5} \mathrm{O}_{10}$. In isolated form, atom of all transition metal elements show nonvanishing magnetic moment following Hund's rule but in solid form only few of them follow that rule to show preserved magnetization. The observation of magnetism in clusters is basically due to large number of oxygen vacancies and moment associated near the surface. ${ }^{33}$ Cluster atom has own characteristics in which valance electrons start to get delocalized and being responsible for magnetism. ${ }^{34}$

\section{CONCLUSIONS}

In conclusion, we have reported the $\left(\mathrm{TiO}_{2}\right)_{n}$ clusters, where $n=2$ to 11 having different geometries. The total ground state energies are found to be inversely proportional to cluster size as the co-ordination number increases with cluster size. The stability of even clusters is higher than odd number clusters because of chemical bonding during formation of the clusters. It is worthwhile to point out here that in the present calculations, we assumed only collinear alignment of spins; so with stable geometry there is induced magnetism and magnetic moments have an oscillatory behavior.

\section{ACKNOWLEDGMENTS}

This work has been supported by the Department of Science and Technology under the research Grant No. SR/ NM/NS-20/2008 and partially supported by LNM Institute of Information Technology, Jaipur, Rajasthan, India.

${ }^{1}$ A. Heller, Science 223, 1141 (1984).

${ }^{2}$ M. Grätzel, Nature 414, 338 (2001).

${ }^{3}$ M. Grätzel, J. Photochem. Photobiol. C 4, 145 (2003).

${ }^{4}$ D. V. Bavykin, J. M. Friedrich, and F. C. Walsh, Adv. Mater. 18, 2807 (2006).

${ }^{5}$ P. L. Gai-Boyes, Catal. Rev. Sci. Eng. 34, 1 (1992).

${ }^{6}$ K. A. Zemski et al., J. Phys. Chem. B 106, 6136 (2002).

${ }^{7}$ G. C. Bond and S. F. Tahir, Appl. Catal. 71, 1 (1991).

${ }^{8}$ H.-J. Zhai and L.-S. Wang, J. Am. Chem. Soc. 129, 3022 (2007).

${ }^{9}$ H. Wu and L.-S. Wang, J. Chem. Phys. 107, 8221 (1997).

${ }^{10}$ W. Yu and R. B. Freas, J. Am. Chem. Soc. 112, 7126 (1990).

${ }^{11}$ Y. Matsuda and E. R. Bernstein, J. Phys. Chem. A 109, 314 (2005).

${ }^{12}$ Y. Matsumoto, Science 291, 854 (2001).

${ }^{13}$ J. M. D. Coey and S. A. Chambers, MRS Bull. 33, 1053 (2008).

${ }^{14}$ J. M. D. Coey, Solid State Mater. Sci. 10, 83 (2006).

${ }^{15}$ D. van Heijnsbergen et al., Phys. Rev. Lett. 83, 4983 (1999).

${ }^{16}$ X. Wei et al., J. Appl. Phys. 105, 07C517 (2009).

${ }^{17}$ L. Chiodo, M. Salazar, A. H. Romero, S. Laricchia, and A. Rubio, J. Chem. Phys. 135, 244704 (2011).

${ }^{18}$ S.-D. Mo and W. Y. Ching, Phys. Rev. B 51, 13023 (1995).

${ }^{19}$ T. Albaret, F. Finocchi, and C. Noguera, Appl. Surf. Sci. 144, 672 (1999).

${ }^{20}$ K. S. Jeong, Ch. Chang, E. Sedlmayr, and D. Sulzle, J. Phys. B 33, 3417 (2000).

${ }^{21}$ Z. W. Qu and G.-J. Kroes, J. Phys. Chem. B 110, 8998 (2006).

${ }^{22}$ A. Hagfeldt, R. Bergstriim, H. O. G. Siegbahn, and S. Lunell, J. Phys. Chem. 97, 12725 (1993).

${ }^{23}$ A. C. Tsipis and C. A. Tsipis, Phys. Chem. Chem. Phys. 1, 4453 (1999).

${ }^{24} \mathrm{~S}$. Li and D. A. Dixon, J. Phys. Chem. A 112, 6646 (2008).

${ }^{25}$ Z. W. Qu and G. J. Kroes, J. Phys. Chem. C 111, 16808 (2007).

${ }^{26}$ W. Zhang, Y. Han, S. Yao, and H. Sun, Mater. Chem. Phys. 130, 196 (2011).

${ }^{27}$ G. Kresse and J. Furthmüller, Phys. Rev. B 54, 11169 (1996).

${ }^{28}$ G. Kresse and D. Joubert, Phys. Rev. B. 59, 1758 (1999).

${ }^{29}$ J. P. Perdew, J. A. Chevary, S. H. Vosko, K. A. Jackson, M. R. Pederson, D. J. Singh, and C. Fiolhais, Phys. Rev. B 46, 6671 (1992).

${ }^{30}$ T. Tsuchiya and J. L. Whitten, J. Chem. Phys. 134, 114701 (2011).

${ }^{31}$ D. J. Mowbray, J. I. Martinez, J. M. García-Lastra, K. S. Thygesen, and K. W. Jacobsen. J. Phys. Chem. C 113, 12301 (2009).

${ }^{32}$ X.-H. Wei et al., IEEE Trans. Magn. 45, 4089 (2009).

${ }^{33}$ H. M. Zhao et al., Physica B 406, 4322 (2011).

${ }^{34}$ J. Bansmanna et al., Surf. Sci. Rep. 56, 189 (2005). 